$n_{1}+1, \cdots$, then the group generated by $i_{1}$ and $H$ contains operators of order $p^{2}$ and the remarks in regard to additional groups apply only to the remaining numbers and to the invariant operators of $H$ which are not commutators. As $i_{1}$ and its conjugates cannot give rise to any group of order $p^{m}$ when $p$ is less than some one of the numbers $n+1$, $n_{1}+1, \cdots$, all the groups of this order which contain $H$ can be readily obtained by the above considerations. It may be observed that this includes all the groups of order $p^{m}$ in which every operator is of order $p$ whenever $m<5$, since every group of order $p^{4}$ contains an abelian subgroup of order $p^{3}$.

STANFORD UNIVERSTTY,

April, 1902.

\title{
A CLASS OF SIMPLY TRANSITIVE LINEAR GROUPS.
}

BY PROFESSOR L. E. DICKSON.

1. In the study of the group defined for any given field by the multiplication table of any given finite group, $*$ it is necessary to discuss the types of simply transitive linear homogeneous groups $G$ whose transformations can be given the form

$$
\begin{gathered}
\xi_{1}^{\prime}=\eta_{1} \xi_{1}, \quad \xi_{2}^{\prime}=\eta_{2} \xi_{1}+\eta_{1} \xi_{2}, \quad \xi_{3}^{\prime}=\eta_{3} \xi_{1}+\alpha \xi_{2}+\eta_{1} \xi_{3}, \\
\xi_{4}^{\prime}=\eta_{4} \xi_{1}+\beta \xi_{2}+\gamma \xi_{3}+\eta_{1} \xi_{4} \\
\xi_{5}^{\prime}=\eta_{5} \xi_{1}+\lambda \xi_{2}+\mu \xi_{3}+\nu \xi_{4}+\eta_{1} \xi_{5}, \cdots .
\end{gathered}
$$

Here $\eta_{1}, \eta_{2}, \eta_{3}, \eta_{4}, \eta_{5}, \cdots$ are the independent parameters, while $\alpha, \beta, \gamma, \lambda, \cdots$ are linear homogeneous functions of the $\eta_{i}$. Burnside $\dagger$ was led to the erroneous conclusion that every such group $G$ is an abelian group. He first concludes that the expression for $\xi_{t}^{\prime}$ contains only the parameters $\eta_{1}, \cdots, \eta_{t}$ and contains $\eta_{t}$ only in the first term $\eta_{t} \xi_{1}$. That this result need not be true is shown by a consideration of the simply transitive group of quaternary transformations

$$
\begin{gathered}
\xi_{1}^{\prime}=\eta_{1} \xi_{1}, \quad \xi_{2}^{\prime}=\eta_{2} \xi_{1}+\eta_{1} \xi_{2}, \quad \xi_{3}^{\prime}=\eta_{3} \xi_{1}+\alpha \xi_{2}+\eta_{1} \xi_{3}, \\
\xi_{4}^{\prime}=\eta_{4} \xi_{1}-\frac{a_{3}}{a_{4}} \alpha \xi_{2}+\eta_{1} \xi_{4},
\end{gathered}
$$

* For the case of a continuous field, Burnside, Proc. Lond. Math. Soc., vol. 29 (1898), pp. 207-224, 546-565; for an arbitrary field, Dickson, Transactions, vol. 3 (1902), pp. 285-301.

† Proc. Lond. Math. Soc., vol. 29, pp. 552-553. 
where $\alpha \equiv a_{2} \eta_{2}+a_{3} \eta_{3}+a_{4} \eta_{4}, a_{4} \neq 0$. Let $Y_{i}$ be the infinitesimal transformation obtained by setting $\eta_{i}=\delta t, \eta_{i}=0$ $(j=1,2,3,4 ; j \neq i)$. Then

$$
\begin{gathered}
Y_{1}=\sum_{i=1}^{4} \xi_{i} \frac{\partial f}{\partial \xi_{1}}, \quad Y_{2}=\xi_{1} \frac{\partial f}{\partial \xi_{2}}+a_{2} \xi_{2} \frac{\partial f}{\partial \xi_{3}}-\frac{a_{3}}{a_{4}} a_{2} \xi_{2} \frac{\partial f}{\partial \xi_{4}}, \\
Y_{3}=\left(\xi_{1}+a_{3} \xi_{2}\right) \frac{\partial f}{\partial \xi_{3}}-\frac{a_{3}{ }^{2}}{a_{4}} \xi_{2} \frac{\partial f}{\partial \xi_{4}}, \\
Y_{4}=a_{4} \xi_{2} \frac{\partial f}{\partial \xi_{3}}+\left(\xi_{1}-a_{3} \xi_{2}\right) \frac{\partial f}{\partial \xi_{4}}, \quad\left(Y_{2} Y_{3}\right)=a_{3} Y_{3}-\frac{a_{3}{ }^{2}}{a_{4}} Y_{4}, \\
\left(Y_{2} Y_{4}\right)=a_{4} Y_{3}-a_{3} Y_{4}, \quad\left(Y_{3} Y_{4}\right)=0, \quad\left(Y_{1} Y_{i}\right)=0 .
\end{gathered}
$$

But the desired result can always be reached by applying a suitable transformation on the variables $\xi_{i}$ and the cogredient transformation on the parameters $\eta_{i}$ (see $\$ \S 4,5$ below). Taking $G$ in this reduced form, Burnside attempts to prove by induction that $G$ is abelian. He supposes that the first $t-1$ equations of $G$ define an abelian group and concludes, from the fact that $G$ is its own parameter group, that the subgroup of $G$ generated by the infinitesimal operations $Y_{1}$, $Y_{2}, \cdots, Y_{t-1}$ corresponding to $\eta_{1}, \eta_{2}, \cdots, \eta_{t-1}$ is abelian. The invalidity of the conclusion is shown by an example. The transformations

$$
\begin{gathered}
\xi_{1}^{\prime}=\eta_{1} \xi_{1}, \quad \xi_{2}^{\prime}=\eta_{3} \xi_{1}+\eta_{1} \xi_{2}, \quad \xi_{3}^{\prime}=\eta_{3} \xi_{1}+\eta_{1} \xi_{3}, \\
\xi_{4}^{\prime}=\eta_{4} \xi_{1}+\left(b_{2} \eta_{2}+b_{3} \eta_{3}\right) \xi_{2}+\left(c_{2} \eta_{2}+c_{3} \eta_{3}\right) \xi_{3}+\eta_{1} \xi_{4}
\end{gathered}
$$

constitute a simply transitive group in reduced form, which is its own parameter group. The first three equations taken alone constitute an abelian group. But $Y_{1}, Y_{2}, Y_{3}$ do not generate a group if $b_{3} \neq c_{2}$. In fact,

$$
\begin{aligned}
Y_{1}=\sum_{i=1}^{4} \xi_{i} \frac{\partial f}{\partial \xi_{i}}, \quad Y_{2}=\xi_{1} \frac{\partial f}{\partial \xi_{2}}+\left(b_{2} \xi_{2}+c_{2} \xi_{3}\right) \frac{\partial f}{\partial \xi_{4}}, \\
Y_{3}=\xi_{1} \frac{\partial f}{\partial \xi_{3}}+\left(b_{3} \xi_{2}+c_{3} \xi_{3}\right) \frac{\partial f}{\partial \xi_{4}}, \quad Y_{4}=\xi_{1} \frac{\partial f}{\partial \xi_{4}}, \\
\left(Y_{1} Y_{2}\right)=0, \quad\left(Y_{1} Y_{3}\right)=0, \quad\left(Y_{2} Y_{3}\right)=\left(b_{3}-c_{2}\right) Y_{4}, \\
\left(Y_{i} Y_{4}\right)=0 \quad(i=1,2,3) .
\end{aligned}
$$

Each of the two preceding examples shows that $G$ need not be abelian.

2. For the case of one variable or the case of two variables, 
the transformations (1) evidently form a simply transitive abelian group. We proceed to consider the cases $n=3,4,5$. The method applies immediately to $n$ variables, but the formulæ are complicated by the necessary use of a triple subscript notation for the coefficients. Set

$$
\begin{array}{ll}
\alpha=\sum_{k=1}^{n} a_{k} \eta_{k}, & \beta=\sum b_{k} \eta_{k}, \quad \gamma=\sum c_{k} \eta_{k}, \\
\lambda=\sum l_{k} \eta_{k}, \quad \mu=\Sigma m_{k} \eta_{k}, & \nu=\sum n_{k} \eta_{k},
\end{array}
$$

where the $a_{k}, \cdots, n_{k}$ are constants for a particular group $G$. The general transformation of $G$ will be designated $T_{\eta}$ or $T_{\eta_{1}, \ldots, \eta_{n}}$. Then the product $T_{\eta^{\prime}} T_{\eta}$ is of the form (1) with $\eta_{i}{ }^{\prime \prime}, \alpha^{\prime \prime}, \beta^{\prime \prime}, \cdots$, in place of $\eta_{i}, \alpha, \beta$, where

$$
\begin{gathered}
\eta_{1}^{\prime \prime}=\eta_{1} \eta_{1}^{\prime}, \quad \eta_{2}^{\prime \prime}=\eta_{2} \eta_{1}^{\prime}+\eta_{1} \eta_{2}^{\prime}, \quad \eta_{3}^{\prime \prime}=\eta_{3} \eta_{1}^{\prime}+\alpha \eta_{2}^{\prime}+\eta_{1} \eta_{3}^{\prime}, \\
\eta_{4}^{\prime \prime}=\eta_{4} \eta_{1}^{\prime}+\beta \eta_{2}^{\prime}+\gamma \eta_{3}^{\prime}+\eta_{1} \eta_{4}^{\prime}, \\
\eta_{5}^{\prime \prime}=\eta_{5} \eta_{1}^{\prime}+\lambda \eta_{2}^{\prime}+\mu \eta_{3}^{\prime}+\nu \eta_{4}^{\prime}+\eta_{1} \eta_{5}^{\prime}, \\
\alpha^{\prime \prime}=\alpha \eta_{1}^{\prime}+\eta_{1} \alpha^{\prime}, \quad \beta^{\prime \prime}=\beta \eta_{1}^{\prime}+\gamma \alpha^{\prime}+\eta_{1} \beta^{\prime}, \quad \gamma^{\prime \prime}=\gamma \eta_{1}^{\prime}+\gamma^{\prime} r_{1}, \\
\lambda^{\prime \prime}=\lambda \eta_{1}^{\prime}+\mu \alpha^{\prime}+\nu \beta^{\prime}+\eta_{1} \lambda^{\prime}, \quad \mu^{\prime \prime}=\mu \eta_{1}^{\prime}+\nu \gamma^{\prime}+\eta_{1} \mu^{\prime}, \\
\nu^{\prime \prime}=\nu \eta_{1}^{\prime}+\eta_{1} \nu^{\prime} .
\end{gathered}
$$

The transformations (1) will form a group if, and only if, $T_{\eta^{\prime}} T_{\eta}=T_{\eta^{\prime \prime}}$, where $\eta_{1}^{\prime \prime}, \cdots, \eta_{n}^{\prime \prime}$ have the values just given, while the relations

$$
\alpha^{\prime \prime}=\sum_{k=1}^{n} a_{k} \eta_{k}^{\prime \prime}, \quad \beta^{\prime \prime}=\sum_{k=1}^{n} b_{k} \eta_{k}^{\prime \prime}, \quad \cdots
$$

reduce to identities in $\eta_{i}$ and $\eta_{i}^{\prime}$. Upon replacing $a^{\prime \prime}, \beta^{\prime \prime}$, $\cdots, \eta_{k}^{\prime \prime}$ by the above values. . Comparing the coefficients of $\eta_{1} \eta_{1}^{\prime}$, we find that $a_{1}, b_{1}, c_{1}, l_{1}, m_{1}, n_{1}$ are zero. For $n=5$, the remaining conditions are

$$
a_{3} a_{k}+a_{4} b_{k}+a_{5} l_{k}=0, \quad a_{4} c_{k}+a_{5} m_{k}=0, \quad a_{5} n_{k}=0,
$$

(5) $\quad c_{3} a_{k}+c_{4} b_{k}+c_{5} l_{k}=0, \quad c_{4} c_{k}+c_{5} m_{k}=0, \quad c_{5} n_{k}=0$,

(6) $\quad n_{3} a_{k}+n_{4} b_{k}+n_{\mathrm{b}} l_{k}=0, \quad n_{4} c_{k}+n_{5} m_{k}=0, \quad n_{5} n_{k}=0$,

(7) $\left\{\begin{array}{c}b_{3} a_{k}+b_{4} b_{k}+b_{5} l_{k}=a_{2} c_{k}, \quad b_{4} c_{k}+b_{5} m_{k}=a_{3} c_{k}, \\ b_{5} n_{k}=a_{4} c_{k}, \quad 0=a_{5} c_{k},\end{array}\right.$ 


$$
\begin{aligned}
& \text { (8) }\left\{\begin{array}{r}
m_{3} a_{k}+m_{4} b_{k}+m_{5} l_{k}=c_{2} n_{k}, \quad m_{4} c_{k}+m_{5} m_{k}=c_{3} n_{k}, \\
m_{5} n_{k}=c_{4} n_{k}, \quad 0=c_{5} n_{k},
\end{array}\right. \\
& \text { (9) }\left\{\begin{array}{rr}
l_{3} a_{k}+l_{4} b_{k}+l_{5} l_{k}=a_{2} m_{k}+b_{2} n_{k}, & l_{4} c_{k}+l_{5} m_{k}=a_{3} m_{k}+b_{3} n_{k}, \\
l_{5} n_{k}=a_{4} m_{k}+b_{4} n_{k}, & 0=a_{5} m_{k}+b_{5} n_{k},
\end{array}\right.
\end{aligned}
$$

where $k$ takes the values $2,3,4,5$.

3. For $n=3$, the $a_{k}$ are the only coefficients to be considered, and the preceding conditions reduce to $a_{3} a_{k}=0(k=2,3)$. Hence $a=a_{2} \eta_{2}$. Then $\eta_{1}{ }^{\prime \prime}, \eta_{2}{ }^{\prime \prime}, \eta_{3}{ }^{\prime \prime}, a^{\prime \prime}$ are symmetrical in $\eta_{i}$ and $\eta_{i}^{\prime}$, so that the group is abelian.

4. For $n=4, l_{k}, m_{k}, n_{k}$ do not occur, so that conditions are

$$
\begin{gathered}
a_{3} a_{k}+a_{4} b_{k}=0, \quad a_{4} c_{k}=0, \quad c_{3} a_{k}+c_{4} b_{k}=0, \quad c_{4} c_{k}=0, \\
b_{3} a_{k}+b_{4} b_{k}=a_{2} c_{k}, \quad b_{4} c_{k}=a_{3} c_{k}, \quad 0=a_{4} c_{k}, \\
(k=2,3,4) .
\end{gathered}
$$

Hence $c_{4}=0$. If either $c_{2}$ or $c_{3}$ is not zero, the conditions reduce to

$$
a_{4}=0, \quad b_{4}=a_{3}=0, \quad c_{8} a_{2}=0, \quad b_{3} a_{2}=a_{2} c_{2} .
$$

If $a_{2} \neq 0$, then $\alpha=a_{2} \eta_{2}, \beta=b_{2} \eta_{2}+b_{3} \eta_{3}, \gamma=b_{3} \eta_{2}$, and $\eta_{j}^{\prime \prime}$, $a^{\prime \prime}, \beta^{\prime \prime}, \gamma^{\prime \prime}$ are symmetrical in $\eta_{i}$ and $\eta_{i}^{\prime}$. The group is therefore abelian. If $a_{2}=0, T_{\eta}$ takes the form (3). The group $G$ is abelian if, and only if, $b_{3}=c_{2}$. If $* b_{3} \neq c_{2}$, the only "ausgezeichnete" infinitesimal transformations are the $e_{1} Y_{1}+e_{4} Y_{4}$.

Let next $c_{2}=c_{3}=0$, so that the conditions are

$$
a_{3} a_{k}+a_{4} b_{k}=0, \quad b_{3} a_{k}+b_{4} b_{k}=0 \quad(k=2,3,4) .
$$

If $a_{4}=0$, then $a_{3}=b_{4}=0, b_{3} a_{2}=0$. If also $a_{2}=0, T_{\eta}$ is of the form (3) with $c_{2}=c_{3}=0$. But if $a_{2} \neq 0$, then $\alpha=a_{2} \eta_{2}$, $\beta=b_{2} \eta_{2}, \gamma=0$, so that the group is abelian. Finally, if $a_{4} \neq 0$, the conditions reduce to the following :

$$
a_{3}+b_{4}=0, \quad a_{3}^{2}+a_{4} b_{3}=0, \quad a_{3} a_{2}+a_{4} b_{2}=0,
$$

whence $\beta=-\alpha a_{3} / \alpha_{4}, \gamma=0$, so that $T_{\eta}$ is of the form (2). The group $G$ is then not abelian (\$1). To bring it to the reduced form, set

* The group is of the type $\left(V^{\prime}\right)$, page 588, Lie-Scheffers, Continuierliche Gruppen. 


$$
x_{3}=a_{3} \xi_{3}+a_{4} \xi_{4}, \quad \zeta_{3}=a_{3} \eta_{3}+a_{4} \eta_{4} .
$$

Then $T_{\eta}$ becomes

$$
\begin{gathered}
\xi_{1}^{\prime}=\eta_{1} \xi_{1}, \quad \xi_{2}^{\prime}=\eta_{2} \xi_{1}+\eta_{1} \xi_{2}, \quad x_{3}^{\prime}=\zeta_{3} \xi_{1}+\eta_{1} x_{3}, \\
\xi_{4}^{\prime}=\eta_{4} \xi_{1}-\frac{a_{3}}{a_{4}}\left(a_{2} \eta_{2}+\zeta_{3}\right) \xi_{2}+\eta_{1} \xi_{4} .
\end{gathered}
$$

Its self-conjugate transformations are the following :

$$
\xi_{1}^{\prime}=\eta_{1}^{\prime} \xi_{1}, \quad \xi_{2}^{\prime}=\eta_{1}^{\prime} \xi_{2}, \quad x_{3}^{\prime}=\eta_{1}{ }^{\prime} x_{3}, \quad \xi_{4}^{\prime}=\eta_{4}{ }^{\prime} \xi_{1}+\eta_{1}^{\prime} \xi_{4} .
$$

The group of transformations (1) on four variables is either abelian or else is one of the types (2) and (3), whose self-conjugate transformations form groups of exactly two parameters.

5. Let next $n=5$. Then $n_{5}=0$. If $a_{5} \neq 0$, the last of the conditions (4) and (7) give $n_{k}=0, c_{k}=0$, and the second condition (4) gives $m_{k}=0$. "Hence $\gamma=\mu=\nu=0$. The first condition (4) gives $a_{3} \alpha+a_{4} \beta+a_{5} \lambda=0$. Set

$$
x_{3}=a_{3} \xi_{3}+a_{4} \xi_{4}+a_{5} \xi_{5}, \quad \zeta_{3}=a_{3} \eta_{3}+a_{4} \eta_{4}+a_{5} \eta_{5} .
$$

Then $x_{3}^{\prime}=\zeta_{3} \xi_{1}+\eta_{1} x_{3}$, so that, by applying a transformation on $\xi_{3}, \xi_{4}, \xi_{5}$ and a transformation on the parameters $\eta_{3}, \eta_{4}, \eta_{5}$, we obtain a transformation (1) with $\alpha=0$.

Let $a_{5}=0, a_{4} \neq 0$. Then $c_{k}=0$, so that $\gamma=0$, and $a_{3} \alpha+a_{4} \beta=0$. Set

$$
x_{3}=a_{3} \xi_{3}+\alpha_{4} \xi_{4}, \quad \zeta_{3}=a_{3} \eta_{3}+a_{4} \eta_{4} .
$$

Then $x_{3}{ }^{\prime}=\zeta_{3} \xi_{1}+\eta_{1} x_{3}$. If $a_{5}=a_{4}=0$, then $a_{3} a_{k}=0$ by (4), so that $a_{3}=0$.

Let $a_{5}=a_{4}=a_{3}=0, c_{5} \neq 0$. Then $n_{k}=0$ by the third equation (5), so that $\nu=0$. Also $c_{3} a_{2} \eta_{2}+c_{4} \beta+c_{5} \lambda=0$, $c_{4} \gamma+c_{5} \mu=0$ by the first and second equations (5). Set

$$
x_{4}=c_{4} \xi_{4}+c_{5} \xi_{5}, \quad \zeta_{4}=c_{4} \eta_{4}+c_{5} \eta_{5} .
$$

Then $x_{4}^{\prime}=\zeta_{4} \xi_{1}-c_{3} \lambda_{2} \eta_{2} \xi_{2}+\eta_{1} x_{4}$. Hence, by applying a transformation on $\xi_{4}, \xi_{5}$ and one on the parameters $\eta_{4}, \eta_{5}$, we obtain a transformation (1) with $\gamma=0$.

Let $a_{5}=a_{4}=a_{3}=0, c_{5}=0$. Then $c_{4}=0$ by (5). If $b_{6} \neq 0$, then $n_{k}=0$ by the third equation (7), so that $\nu=0$. By the first and second equations (7),

$$
\left(b_{3}-c_{2}\right) a_{2} \eta_{2}-a_{2} c_{3} \eta_{3}+b_{4} \beta+b_{5} \lambda=0, \quad b_{4} \gamma+b_{5} \mu=0 .
$$


Hence

$$
\begin{gathered}
x_{4}^{\prime}=\zeta_{4} \xi_{1}+\left[\left(c_{2}-b_{3}\right) a_{2} \eta_{2}+a_{2} c_{3} \eta_{3}\right] \xi_{2}+\eta_{1} x_{4}, \\
x_{4} \equiv b_{4} \xi_{4}+b_{5} \xi_{5}, \zeta_{4} \equiv b_{4} \eta_{4}+b_{5} \eta_{5} .
\end{gathered}
$$

We may therefore take $b_{5}=0$. Then $b_{4} b_{k}=0$ by the first equation (7), so that $b_{4}=0$. Then $m_{5}=0$ by the second equation (8). Hence $l_{\overline{0}}=0$ by the first equation (9). Hence $T_{\eta}$ becomes

$$
\begin{aligned}
& \xi_{1}^{\prime}=\eta_{1} \xi_{1}, \quad \xi_{2}^{\prime}=\eta_{2} \xi_{1}+\eta_{1} \xi_{2}, \quad \xi_{3}^{\prime}=\eta_{3} \xi_{1}+a_{2} \eta_{2} \xi_{2}+\eta_{1} \xi_{3}, \\
& \xi_{4}^{\prime}=\eta_{4} \xi_{1}+\left(b_{2} \eta_{2}+b_{3} \eta_{3}\right) \xi_{2}+\left(c_{2} \eta_{2}+c_{3} \eta_{3}\right) \xi_{3}+\eta_{1} \xi_{4}, \\
& \xi_{5}^{\prime}=\eta_{5} \xi_{1}+\left(l_{2} \eta_{2}+l_{3} \eta_{3}+l_{4} \eta_{4}\right) \xi_{2}+\left(m_{2} \eta_{2}+m_{3} \eta_{3}+m_{4} \eta_{4}\right) \xi_{3} \\
& \quad+\left(n_{2} \eta_{2}+n_{3} \eta_{3}+n_{4} \eta_{4}\right) \xi_{4}+\eta_{1} \xi_{5} .
\end{aligned}
$$

Since the group is now in its reduced form, it contains the self-conjugate transformations, in which $\eta_{1}{ }^{\prime}$ and $\eta_{5}{ }^{\prime}$ are arbitrary, $\eta_{1}^{\prime} \neq 0$,

$$
\xi_{i}^{\prime}=\eta_{1}^{\prime} \xi_{i}(i=1,2,3,4), \quad \xi_{5}^{\prime}=\eta_{5}^{\prime} \xi_{1}+\eta_{1}^{\prime} \xi_{5} .
$$

6. The conditions (4)-(9) on $T_{\eta}$ in its reduced form are $c_{3} a_{2}=0, \quad n_{3} a_{2}+n_{4} b_{2}=0, \quad n_{4} b_{3}=0, \quad n_{4} c_{2}=0, \quad n_{4} c_{3}=0$, $b_{8} a_{2}=a_{2} c_{2}, \quad m_{3} a_{2}+m_{4} b_{2}=c_{2} n_{2}, \quad m_{4} b_{8}=c_{2} n_{3}, \quad m_{4} c_{2}=c_{8} n_{2}$, $m_{4} c_{8}=c_{3} n_{3}, \quad l_{8} a_{2}+l_{4} b_{2}=a_{2} m_{2}+b_{2} n_{2}, \quad l_{4} b_{3}=a_{2} m_{3}+b_{2} n_{3}$,

$$
0=a_{2} m_{4}+b_{2} n_{4}, \quad l_{4} c_{2}=b_{3} n_{2}, \quad l_{4} c_{3}=b_{8} n_{8} .
$$

If $n_{4} \neq 0$, then $b_{3}=c_{2}=c_{3}=0, n_{3} a_{2}+n_{4} b_{2}=0$. Set

$$
x_{4}=n_{8} \xi_{3}+n_{4} \xi_{4}, \quad \zeta_{4}=n_{8} \eta_{8}+n_{4} \eta_{4} .
$$

Then $x_{4}^{\prime}=\zeta_{4} \xi_{1}+\eta_{1} x_{4}$. Hence by introducing $x_{4}$ in place of $\xi_{4}$, and $\zeta_{4}$ in place of $\eta_{4}, T_{\eta}$ retains its reduced form and has $b_{2}=b_{8}=c_{2}=c_{8}=0$. Then

$$
n_{3} a_{2}=0, \quad m_{3} a_{2}=0, \quad l_{3} a_{2}=m_{2} a_{2}, \quad m_{4} a_{2}=0,
$$

are the only further conditions.

If $a_{2} \neq 0$, we obtain the transformation

$$
\begin{gathered}
\xi_{1}^{\prime}=\eta_{1} \xi_{1}, \quad \xi_{2}^{\prime}=\eta_{2} \xi_{1}+\eta_{1} \xi_{2}, \quad \xi_{3}^{\prime}=\eta_{3} \xi_{1}+a_{2} \eta_{2} \xi_{2}+\eta_{1} \xi_{3}, \\
\xi_{4}^{\prime}=\eta_{4} \xi_{1}+\eta_{1} \xi_{4}, \\
\xi_{5}^{\prime}=\eta_{5} \xi_{1}+\left(l_{2} \eta_{2}+l_{3} \eta_{3}+l_{4} \eta_{4}\right) \xi_{2}+l_{3} \eta_{2} \xi_{3}+\left(n_{2} \eta_{2}+n_{4} \eta_{4}\right) \xi_{4}+\eta_{1} \xi_{5} .
\end{gathered}
$$


It is readily verified that these transformations form a group with the parameters $\eta_{1} \cdots, \eta_{5}$, whatever be the values of $a_{23} l_{2}, l_{3}, l_{4}, n_{2}, n_{4}$. The expressions for $\eta_{i}^{\prime \prime}(i=1,2,3,4)$ are symmetric in $\eta_{i}$ and $\eta_{i}^{\prime}$, but that for $\eta_{5}^{\prime \prime}$ is symmetric if, and only if, $n_{2}=l_{4}$. In the latter case only, the group is abelian. For $n_{2} \neq l_{4}$, a transformation will belong also to the reciprocal group if, and only if, $\eta_{2}=\eta_{4}=0$. Hence the subgroup of self-conjugate transformations has three arbitrary parameters $\eta_{1}, \eta_{3}, \eta_{5}$.

But, if $a_{2}=0$, the conditions (10) become identities. Hence the transformations

$$
T_{\eta} \text { with } a_{2}=b_{2}=b_{3}=c_{2}=c_{3}=0, n_{4} \neq 0,
$$

form a group, whatever be the values of $l_{i}, m_{i}, n_{i}$. It is abelian if, and only if, $m_{2}=l_{3}, n_{2}=l_{4}, n_{3}=m_{4}$. A selfconjugate transformation must have

$$
\begin{gathered}
\eta_{2}=\eta_{3}=0, \quad \text { if } m_{2} \neq l_{3} ; \quad \eta_{2}=\eta_{4}=0, \text { if } n_{2} \neq l_{4} ; \\
\eta_{3}=\eta_{4}=0, \quad \text { if } n_{3} \neq m_{4} .
\end{gathered}
$$

If $G$ is not abelian, the subgroup of its self-conjugate transformations has two or three arbitrary parameters.

Let next $n_{4}=0$. If $a_{2} \neq 0$, the conditions are

$$
\begin{gathered}
c_{3}=n_{3}=m_{4}=0, \quad b_{3}=c_{2}, \quad\left(l_{3}-m_{2}\right) a_{2}=\left(n_{2}-l_{4}\right) b_{2}, \\
m_{3} a_{2}=c_{2} n_{2}=l_{4} c_{2} .
\end{gathered}
$$

If also $n_{2}=l_{4}$, so that $l_{3}=m_{2}$, then $\eta_{j}^{\prime \prime}(j=1, \cdots, 5)$ is symmetric in $\eta_{i}$ and $\eta_{i}^{\prime}$ and the group is abelian. If $n_{2} \neq l_{4}$, and $l_{3}=m_{2}$, then $c_{2}=0, b_{2}=0, m_{3}=0$, so that

$$
\begin{gathered}
\xi_{4}^{\prime}=\eta_{4} \xi_{1}+\eta_{1} \xi_{4}, \xi_{5}^{\prime}=\eta_{5} \xi_{1}+\left(l_{2} \eta_{2}+l_{3} \eta_{3}+l_{4} \eta_{4}\right) \xi_{2} \\
+l_{3} \eta_{2} \eta_{3}+n_{2} \eta_{2} \xi_{4}+\eta_{1} \xi_{5},
\end{gathered}
$$

with the restrictions $a_{2} \neq 0, n_{2} \neq l_{4}$. The self-conjugate transformations have $\eta_{2}=\eta_{4}=0, \eta_{1}, \eta_{3}, \eta_{5}$ arbitrary. If $n_{2} \neq l_{4}$, and $l_{3} \neq m_{3}$, then $c_{2}=m_{3}=0$, and the self-conjugate transformations have $\eta_{2}=\eta_{3}=\eta_{4}=0, \eta_{1}$ and $\eta_{3}$ arbitrary.

Let next $n_{4}=a_{2}=0$. The conditions are

$$
\begin{aligned}
& m_{4} b_{2}=c_{2} n_{2}, \quad m_{4} b_{3}=c_{2} n_{3}, \quad m_{4} c_{2}=c_{3} n_{2}, \quad\left(m_{4}-n_{3}\right) c_{3}=0 \\
& l_{4} b_{3}=b_{2} n_{3}, \quad l_{4} c_{2}=b_{3} n_{2}, \quad l_{4} c_{3}=b_{3} n_{3}, \quad\left(l_{4}-n_{2}\right) b_{2}=0 .
\end{aligned}
$$

The transformations form an abelian group if, and only if

$$
b_{3}=c_{2}, \quad l_{3}=m_{2}, \quad l_{4}=n_{2}, \quad m_{4}=n_{3}, \quad n_{3} c_{2}=c_{3} n_{2}, \quad n_{3} b_{2}=c_{2} n_{2} .
$$


The form of the general transformation can be simplified by applying a transformation on $\xi_{2}, \xi_{3}$, and the cogredient transformation on $\eta_{2}, \eta_{3}$, and similarly a transformation on $\xi_{4}, \xi_{5}$ and one on $\eta_{4}, \eta_{5}$.

7. The argument of Burnside, 1. c., $\$ 6$, page 553, is faulty. It does not show that $\nu=\mu$, but does prove that $\nu$ is a multiple of $\mu$. In view of the work of Frobenius and that of Molien, the theorem in question is true.

The University of Chicago, May 12, 1902.

\section{ERRORS IN LEGENDRE'S TABLES OF LINEAR DIVISORS.}

BY DR. D. N. LEHMER.

Some years ago an error in Legendre's Tables of Linear Forms came to my notice. Another was found recently by members of my class, and as this error was left without correction in the later editions I determined to make a careful computation of the whole set. I was surprised to find the list of errors so long. The importance of these tables for many investigations makes it desirable that all these corrections be noted. I have also compared results with the tables in Tshebyshef's Theorie der Congruenzen, Berlin, 1889. Most of the errors in Legendre's work have been carried over uncorrected into these tables.

I. Under the form $t^{2}-29 u^{2}$ the form $116 x+3$ should read $116 x+7$. This error was corrected in the fourth edition (1900), which is a copy of the edition of 1830 .

II. Under the form $t^{2}-38 u^{2}$ the form $152 x+129$ should read $152 x+131$. Not corrected in the fourth edition nor in Tshebyshef.

III. Under the form $t^{2}-43 u^{2}$ the form $172 x+147$ should read $172 x+137$. Not corrected in the fourth edition nor in Tshebyshef.

IV. Under $t^{2}-51 u^{2}$ there are two forms $204 x+13$. The second of these should read $204 x+31$. This error is in the fourth edition but not in the first (1797).

$\mathrm{V}$. Under $t^{2}-61 u^{2}$ there are so many errors that I will give the correct list: $244 x+1,3,5,9,13,15,19,25,27$, $39,41,45,47,49,57,65,73,75,77,81,83,95,97,103$, $107,109,113,117,119,121,123,125,127,131,135,137$, $141,147,149,161,163,167,169,171,179,187,195,197$, $199,203,205,217,219,225,229,231,235,239,241,243$. The 